\title{
Stake effects on ambiguity attitudes for gains and losses
}

\author{
Ranoua Bouchouicha ${ }^{1}$ - Peter Martinsson ${ }^{2,3}$. \\ Haileselassie Medhin $^{2,4}$. Ferdinand M. Vieider ${ }^{5,6}$
}

Published online: 5 January 2017

(C) The Author(s) 2017. This article is published with open access at Springerlink.com

\begin{abstract}
We test the effect of stake size on ambiguity attitudes. Compared to a baseline condition, we find subjects to be more ambiguity seeking for small-probability gains and large-probability losses under high stakes. They are also more ambiguity averse for large-probability gains and small-probability losses. We trace these effects back to stake effects on decisions under risk (known probabilities) and uncertainty (unknown probabilities). For risk, we replicate previous findings. For uncertainty, we find an increase in probabilistic insensitivity under high stakes that is driven by increased uncertainty aversion for large-probability gains and for small-probability losses.
\end{abstract}

Keywords Monetary stakes · Ambiguity aversion · Risk attitudes

Financial support from the University of Munich and the Swedish Research Council is gratefully acknowledged. We are grateful for the support given us by the Ethiopian Development Research Institute.

$凶 \quad$ Ferdinand M. Vieider

f.vieider@reading.ac.uk

1 Henley School of Business, University of Reading, Reading, UK

2 Department of Economics, University of Gothenburg, Gothenburg, Sweden

3 Linköping University, Linköping, Sweden

4 Ethiopian Development Research Institute, Addis Ababa, Ethiopia

5 Department of Economics, University of Reading, Reading, UK

6 WZB Berlin Social Science Center, Berlin, Germany 


\section{Introduction}

Some form of uncertainty is involved in most important decision-making processes, and uncertainty about the future may, indeed, be considered inherent to the human condition. It is thus not surprising that how people react to such uncertainties has received considerable attention in decision theory. Most empirical investigations have, however, concentrated on the special case of risk, where objective probabilities are known. While this case may be a good representation of some decision situations, such as roulette wheels or many medical decision problems (for which precise probabilities can be derived from a large number of past observations), the case of uncertainty proper, for which no objectively derivable probabilities are available, is likely to be much more important in praxis.

Notwithstanding its importance in the real world, such uncertainty has until recently mostly been studied in opposition to behavior under risk, and even so mostly for a restricted class of 50-50 gambles over gains (see Trautmann and van de Kuilen (2015), for an overview). The phenomenon by which people tend to prefer outcome generating processes characterized by known probabilities (risk) over outcome generating processes with vague or unknown probabilities (uncertainty) which are normatively equivalent under subjective expected utility theory (Savage 1954) is known as ambiguity aversion (Ellsberg 1961). In addition to the challenges it creates for subjective expected utility, ambiguity aversion has been deemed to be an important determinant of a variety of real-world decisions, including the home bias (Kilka and Weber 2000), reluctance to vaccinate (Ritov and Baron 1990), and the preference for established brands over new ones (Muthukrishnan et al. 2009), to name but a few.

Only recently has a more systematic way of measuring ambiguity attitudes been developed, which allows for measurements over the whole probability spectrum and for less artificial types of uncertainty (Abdellaoui et al. 2011, 2005; Fox and Tversky 1998; Kilka and Weber 2000; Maafi 2011; van de Kuilen and Wakker 2011). Given the recency of these developments, many issues remain to be explored. This paper specifically investigates whether ambiguity attitudes are affected by the magnitude of the monetary outcomes at stake, a problem on which no evidence exists to date. Ambiguity attitudes are thereby estimated for different stake levels over the whole probability space. In addition, we can trace any stake effects we find for ambiguity back to its constituent parts - stake effects for risk and stake effect for uncertaintyand thus determine what underlying patterns drive stake effects for ambiguity. Contrary to most of the literature on stake effects under risk, we use a between-subjects design which avoids potential contrast effects resulting from the direct contraposition of high and low stake decisions (Greenwald 1978; Read 2005).

The issue of stake effects on ambiguity and uncertainty attitudes is important to the extent that real-world decisions involving more or less well-known probabilities may concern a diverse spectrum of outcomes, ranging from relatively trivial stakes to extremely high ones. Any differences in ambiguity attitudes dependent on stakes can thus be informative about this type of behavior. Furthermore, possible stake effects under uncertainty provide information on the extent to which results obtained with low stakes in the laboratory can be generalized to decisions with non-trivial stakes in the field, or on how any such generalizations might need to be adjusted for differences 
in stakes. Since we elicit certainty equivalents (CEs) for a number of prospects with different probabilities, we can analyze how such a stake effect manifests itself over the whole probability space. Furthermore, we present evidence for both gains and losses. This may be interesting for analyzing decisions, e.g., on the stock market, which can be characterized as decisions under true uncertainty, and which have been linked to ambiguity aversion

In contrast to uncertainty, the effect of different stake sizes has been studied extensively for the case of risk, especially for gains. In an experiment conducted in rural India, Binswanger (1980) showed that risk aversion increases in stake levels. Kachelmeier and Shehata (1992) ran high stakes experiments in China, finding that risk seeking for small probabilities was reduced relative to smaller stakes, a result that was replicated by Lefebvre et al. (2010) in France using a between subjects design. Holt and Laury (2002) supplied further evidence on how subjects become more risk averse as stakes increase, and Fehr-Duda et al. (2010) showed with Chinese subjects how, in a prospect theory framework, such stake effects will affect the probability weighting function as well as the utility function, thus violating the separability precept by which attitudes towards money are supposed to be reflected purely in utility (although this may, to some extent, depend on the functional form of utility - see Scholten and Read (2014), and Bouchouicha and Vieider (2016)).

Stake effects are less well understood for risky losses. Hogarth and Einhorn (1990) found no effect of such stake variations on losses. Etchart-Vincent (2004) tested different hypothetical stake levels against each other, and found only slight evidence for increased risk aversion under high stakes. Bosch-Domènech and Silvestre (2006), on the other hand, found large increases in risk aversion for both small and large probabilities of losses as stakes increased. Fehr-Duda et al. (2010) found inconclusive effects in the loss domain. Moreover, there exist several papers investigating the issue of whether hypothetical choice differs from choices for real money under losses (Etchart-Vincent and L'Haridon 2011; Laury et al. 2009; Schoemaker 1990; Vieider 2011), which is, however, quite a different issue. We provide some additional evidence on the effect of stake sizes for decisions under risk in the loss domain.

We conducted an experiment in Ethiopia where we compare a baseline condition, providing already relatively high stakes, to a high stake condition in which all monetary amounts are doubled in a clean between subjects design. For gains, high stakes cause increases in ambiguity seeking for small probabilities and increases in ambiguity aversion for large probabilities relative to the baseline. This means that under high stakes, we observe more extreme jumps at the endpoints of the probability scale, resulting in reduced sensitivity to probabilistic change in the intermediate ranges. We next trace this effect back to stake effects for risk and uncertainty. For known probabilities, we replicate the well-known finding of risk aversion increasing in stake size - an effect that is uniform over the probability space. For unknown probabilities, we find uncertainty aversion to increase in stake sizes just as for risk. This effect, however, happens entirely through increased uncertainty aversion for large probabilities with uncertainty attitudes for small probabilities remaining unchanged, thus resulting in reduced probabilistic sensitivity for intermediate probabilities. The effect found under ambiguity is thus driven mainly by stake effects in decisions under uncertainty. This pattern is mirrored for losses. In the baseline condition, we find a pattern of ambiguity 
aversion for low probability levels and ambiguity seeking for high probability levels. Under high stakes, we again find an accentuation of these patterns at the endpoints of the probability scales, with subjects being more ambiguity averse for small probabilities and more ambiguity seeking for large probabilities. Once again, this pattern is driven by more extreme uncertainty attitudes at the endpoints of the probability scale, while we find no effect of stakes on risk attitudes.

\section{Experiment: setup and method}

Subjects. A total of 157 subjects were recruited by the authors at Addis Ababa University, in Ethiopia, on the campus of Economics, Management, and Information Sciences. The experiment was conducted in English - the teaching language of the university - and one of the authors, who conducted the experiments and is fluent in Amharic (the local language), provided additional explanations where needed. A total of 12 subjects had to be excluded, because they manifestly did not understand the task or did not respond to all questions. The average age was 21.1 years, and $73 \%$ of subjects were male.

Tasks. We elicited certainty equivalents (CEs) for simple binary prospects through choice lists. This procedure involved making subjects choose between a prospect and different sure amounts of money increasing in steps of 3 Birr from the low amount in the prospect to the high amount of the prospect, with the amounts being mirrored for losses, which took place from an endowment. The tasks are shown in Table 1, and the complete instructions can be found in the online appendix or on www.ferdinandvieider. com/instructions.html. Subjects were told that there were no right or wrong answers and that we were only interested in their preferences. They were also told, however, that we were interested in the point where they would switch from preferring the prospect to preferring the sure amount (the opposite for losses), and that they should not switch back and forth between prospect and sure amount. For unknown probabilities, the elicitation of CEs has the advantage of producing a measure of the strength of preferences, while avoiding biases that result from the elicitation of willingness to

Table 1 Decision tasks, amounts in Ethiopian Birr

\begin{tabular}{|c|c|c|c|}
\hline \multicolumn{2}{|l|}{ Baseline } & \multicolumn{2}{|l|}{ High stakes } \\
\hline Gains & Losses & Gains & Losses \\
\hline$(1 / 8: 120 ; 0)$ & $(1 / 8:-120 ; 0)$ & $(1 / 8: 240 ; 0)$ & $(1 / 8:-20 ; 0)$ \\
\hline$(1 / 8: 120 ; 30)$ & $(1 / 8:-120 ;-30)$ & $(1 / 8: 240 ; 60)$ & $(1 / 8:-240 ;-60)$ \\
\hline$(2 / 8: 120 ; 0)$ & $(2 / 8:-120 ; 0)$ & $(2 / 8: 240 ; 0)$ & $(2 / 8:-240 ; 0)$ \\
\hline$(3 / 8: 120 ; 0)$ & $(3 / 8:-120 ; 0)$ & $(3 / 8: 240 ; 0)$ & $(3 / 8:-240 ; 0)$ \\
\hline$(5 / 8: 120 ; 0)$ & $(5 / 8:-120 ; 0)$ & $(5 / 8: 240 ; 0)$ & $(5 / 8:-240 ; 0)$ \\
\hline$(6 / 8: 120 ; 0)$ & $(6 / 8:-120 ; 0)$ & $(6 / 8: 240 ; 0)$ & $(6 / 8:-240 ; 0)$ \\
\hline$(7 / 8: 120 ; 0)$ & $(7 / 8:-120 ; 0)$ & $(7 / 8: 240 ; 0)$ & $(7 / 8:-240 ; 0)$ \\
\hline$(7 / 8: 120 ; 30)$ & $(7 / 8:-120 ;-30)$ & $(7 / 8: 240 ; 60)$ & $(7 / 8:-240 ;-60)$ \\
\hline
\end{tabular}


pay or willingness to accept measures (Trautmann et al. 2011). The CEs were elicited using paper and pencil and keeping the order of the tasks constant throughout the experiment. Risky gains were followed by uncertain gains. Once this first part of the experiment was over, subjects received a second set of instructions concerning the loss part. Again, CEs for risky losses were elicited first and then CEs for uncertain losses were elicited. While such a fixed ordering of tasks may induce order effects, we felt that such effects were preferable to the increased noise levels that were likely to result from a randomization of the order in which the decision tasks were presented. Furthermore, a large-scale pilot revealed that no such order effects occurred in our design. Losses took place from an endowment. Etchart-Vincent and L'Haridon (2011) tested whether such losses from endowment would result in different effects from actual losses and found no such differences. They also found no order effect for losses being administered before or after gains. Risky prospects were represented using eight consecutively numbered balls in an urn. Uncertain prospects, implemented through Ellsberg-type urns, were also represented through eight numbered balls, and subjects were told that while there were always eight balls in the vague-probability urn just as in the risky one, it was possible that some numbers may be absent, while others would occur repeatedly (the names risky and uncertain were not used in the experiment, the urns were called transparent and opaque instead). This procedure follows closely the one used by Abdellaoui et al. (2011), except that we used numbers instead of colors to be able to print instructions in black and white.

Treatments and payoffs. 83 subjects participated in the baseline condition and 62 in the high stake condition in a between-subjects design. The gender ratio did not significantly differ between treatments $(z=0.336, p=0.73)$. While within-subject designs have often been employed for stake comparisons in the literature, we chose this alternative to avoid contrast effects that can potentially result in experimenter demand effects in within-subject designs (Greenwald 1978; Read 2005). Running the experiment in Ethiopia allowed us to offer non-trivial payoffs to the subjects. The monetary prize to be won in the baseline condition was 120 Birr. This is the PPP equivalent of $€ 20$ and it corresponds to about $20-25 \%$ of the total monthly expenditure of the median participant in the experiment. In the high stake condition, all monetary amounts were doubled. While this may be considered a relatively modest increase in stake sizes compared to other manipulations in the literature, this also means that any effects found are all the more notable. Notice also that, while some previous studies on stake sizes used higher stake differences between treatments, they typically did so from a much lower baseline. ${ }^{1}$ We chose a higher baseline on purpose to determine whether choices would further change as compared to typical experimental payoffs, even when the latter are already on the high side. At the end of the experiment, one of the decisions was randomly selected for real pay - the standard procedure in this kinds of experiment. Estimation and analysis. Stake effects can be difficult to analyze within a certain decision model. For instance, prospect theory (Kahneman and Tversky 1979; Tversky and Kahneman 1992) — the prevalent descriptive theory of decision-making under risk

\footnotetext{
1 For instance, Holt and Laury (2002) increased their stakes 20-fold and Lefebvre et al. (2010) by factors of four and ten. The baseline in the first study, however, was about $\$ 2$, and it was about $€ 4$ in the second.
} 


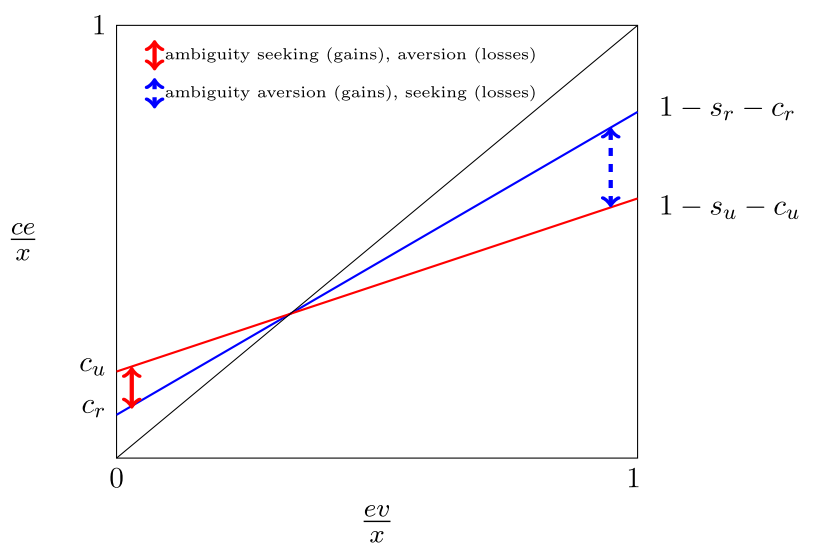

Fig. 1 Illustration of indices

and uncertainty today (Starmer 2000; Wakker 2010)—represents decisions through utility and probability weighting functions, which both contribute to risk attitudes. This, however, means that any effects of stakes on risk attitudes may affect both utility and probability weighting and, in some cases, produce opposite effects on the two, such that the final effect on risk attitudes may be unclear (Vieider 2012). It is thus convenient to employ some indices that summarize the information over the probability space, so as to systematically organize the information derived from several certainty equivalents, without recurring to any particular theory to organize the results. To this end, we estimate the best linear fit for the CEs by maximum-likelihood estimation at the individual level:

$$
\frac{c e_{i}}{x}=c+s \times \frac{e v_{i}}{x}
$$

where $X$ is the highest outcome or prize to be won in the prospect and $i$ is an index designating the particular prospect at hand. This regression line is estimated separately for gains and losses, and a number of indices are derived from it. The indices are illustrated in Fig. 1. A positive intercept, $c$, indicates a certainty equivalent for small probabilities that is larger than the expected value (EV) of the prospect in absolute terms (indicating risk seeking for gains and risk aversion for losses), and a slope, s, smaller than 1 indicates that CEs increase less than linearly in the EV of the prospect and is thus an index of the sensitivity of CEs to changes in probability. We estimate these indices separately for risk, designated by the subscript $r$, and uncertainty, designated by the subscript $u$. Ambiguity attitudes are then quantified by the difference between the indices obtained under risk and under uncertainty, as indicated by the arrows.

In addition to the intercept and the slope, we use two more indices to make the exposition more intuitive (see Abdellaoui et al. 2010, for a discussion). One is the anti-intercept, that is the distance of the regression line from 1 at $p=1$, which is given by $1-s-c$, and a positive value of which indicates risk aversion for gains or risk seeking for losses. Finally, we can construct a global measure of average attitudes over the probability space by subtracting the intercept from the anti-intercept, $1-s-2 c$ 
(Abdellaoui et al. 2011). This index takes the value of zero under risk neutrality, is positive if there is a tendency towards risk aversion (risk seeking for losses), and is negative if the general tendency goes towards risk seeking (risk aversion for losses). While risk attitudes are fully characterized by any two of these four measures, it may sometimes be convenient to present results for all four of them. Notice that how these indices are formally equivalent to the indices employed by Abdellaoui et al. (2011) to analyze neo-additive weighting functions (Chateauneuf et al. 2007). Notice also, however, that, in our case, these indices do not represent attributes of a probability weighting function, but a linear best fit for our non-parametric data.

Noise and violations of stochastic dominance. Overall, noise levels in the data were rather high. Such high noise levels are not unusual for developing countries (L'Haridon and Vieider 2016). Some subjects systematically violated the first-order stochastic dominance, which resulted in a negative slope of the regression line described above. Violations of stochastic dominance are common even amongst highly educated subjects in the West (Birnbaum 1999). Since such preferences violate basic principles of rationality, we excluded subjects who had a negative slope for any of the four decision domains involved-risky gains, uncertain gains, risky losses, or uncertain losses. This brought us to eliminate 13 subjects in the high stake condition and 19 subjects in the baseline condition. The results reported below do not change qualitatively if these subjects are included in the analysis.

Statistics. The indices developed above constitute a convenient tool for statistical analysis, since they can be estimated at the individual level. Graphical displays use median parameter estimates throughout. All statistics reported are Mann-Whitney tests where two different variables or indices are compared, and Wilcoxon signedrank tests where indices or variables are compared to a normative benchmark. All $p$-values reported are two-sided, unless otherwise specified.

Hypotheses. We hypothesize that risk aversion will increase in stake size for all probability levels for gains. For losses, we hypothesize that stakes will have no effect on risk attitudes. Since there is no evidence so far on stake size effects on decisions under uncertainty, our hypotheses are by necessity more tentative. We anticipate the same qualitative effects as for risk, although quantitatively the effects may be weaker given lower levels of sensitivity under uncertainty. Finally, we attempt no direct hypotheses about effects on ambiguity attitudes, since the latter depend on the quantitative differences between the effects on risk and on uncertainty, for which we have no clear predictions.

\section{Results}

\subsection{Ambiguity attitudes for gains}

We start by looking at stake effects on ambiguity attitudes, represented in Fig. 2, with the probability of winning represented on the $\mathrm{x}$-axis and ambiguity aversion represented on the $y$-axis. Ambiguity attitudes are now represented by the area between each of the two lines and the $\mathrm{x}$-axis (which can be seen as representing normalized 


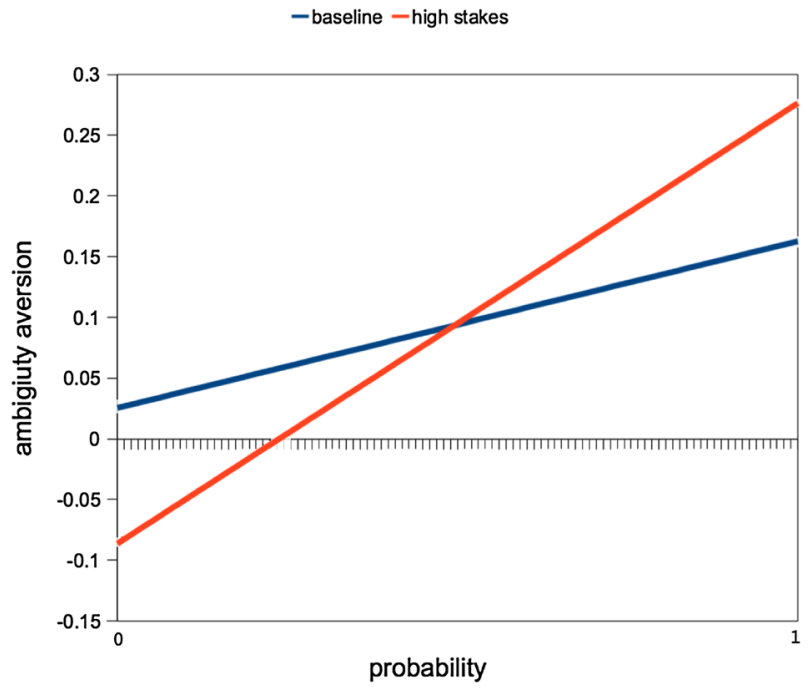

Fig. 2 Ambiguity aversion for gains by stake size

risk attitudes, since ambiguity attitudes are defined as the difference between risk and uncertainty attitudes), with areas above the $\mathrm{x}$-axis representing ambiguity aversion and areas below the $\mathrm{x}$-axis representing ambiguity seeking (i.e., the distance between risk and uncertainty attitudes shown by the solid arrow in Fig. 1 is now given by a negative distance from zero, and the distance captured by the dashed arrow is given by a positive distance from zero, representing ambiguity aversion for gains).

In the baseline condition, we observe ambiguity aversion over the whole probability space, although we cannot exclude the null hypothesis of an intercept equal to zero $(z=1.18, p=0.24)$, indicating no ambiguity aversion for small probabilities. There is, however, clear ambiguity aversion for large probabilities, with an anti-intercept significantly larger than zero $(z=4.37, p<0.001)$. There is also clear ambiguity aversion at the global level, as indicated by a significantly positive index of global attitudes $(z=5.18, p<0.001)$. While for small probabilities, we find subjects to be marginally more ambiguity seeking under high stakes relative to the baseline $(z=1.73, p=0.083)$, subjects are considerably more ambiguity averse for large probabilities under high stakes compared to the baseline $(z=3.09, p=0.002)$. While the opposing trends at the two endpoints cancel out at the global level, so that there is no difference in terms of global or average ambiguity attitudes $(z=1.03, p=0.31)$, there is a considerable change in the slope indicating a movement towards more extreme attitudes at the two endpoints of the probability $\operatorname{spectrum}(z=3.03, p=0.002){ }^{2}$

\footnotetext{
2 Notice that how a decrease in probabilistic sensitivity is now indicated by a steeper line. This is due to the fact that the line represents sensitivity under risk minus sensitivity under uncertainty. The steeper line that we find under high stakes may thus derive either from decreased sensitivity under uncertainty keeping sensitivity for risk constant or increased sensitivity under risk keeping sensitivity under uncertainty constant (or a combination of both). This issue is discussed below.
} 


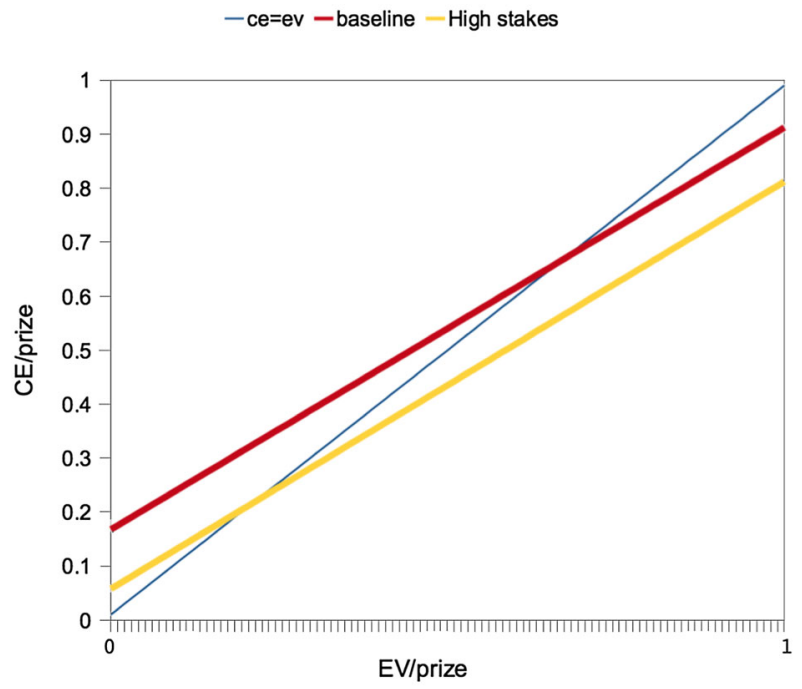

Fig. 3 Ambiguity aversion for gains by stake size

To understand what is driving the above results, we must look at the effect of the stake variation on risk and uncertainty attitudes separately. Figure 3 shows stake effects for decisions involving known probabilities (i.e., risk) for gains. The first thing that is apparent from the figure is that there is a considerable amount of risk seeking in the baseline condition. Indeed, we find risk seeking to be the dominant pattern in the baseline condition $(z=3.14, p=0.002)$. High levels of risk seeking are actually less rare than supposed so far-for a discussion and a worldwide comparison of risk attitudes, see L'Haridon and Vieider (2016). ${ }^{3}$ As to the effect of increased stakes, it is clearly apparent that the regression line shifts downward in a parallel fashion as stakes increase. There is less risk seeking for small probabilities under larger stakes $(z=2.78, p=0.006)$, more risk aversion for large probabilities $(z=1.80, p=0.073)$, and hence also more risk aversion on a global level $(z=3.37, p=0.001)$. The slope of the line, indicating insensitivity to probabilistic change, is not affected by stake size $(z=0.13, p=0.89)$. These results thus replicate the previous findings in the literature.

Figure 4 shows the equivalent regression lines for the case of unknown probabilities for gains. In the baseline condition, we find that for small (vague) probabilities, our subjects are uncertainty seeking, with a certainty equivalent that is higher than the expected value of the prospect $(z=6.58, p<0.001)$. For large probabilities, we observe uncertainty aversion $(z=5.54, p<0.001)$. The slope of the regression line is

\footnotetext{
3 High levels of risk seeking have also been found before in Ethiopia (Doerr et al. 2011), as well as other East African countries (Henrich and McElreath 2002). Vieider et al. (2015b) show that these attitudes are stable across different sample in Addis Ababa, and Vieider et al. (2016) furthermore show that the results here reported carry over to a representative sample of the rural population of the Ethiopian highlands (see, however, also Akay et al. 2012, for a contrary finding in a more geographically contained farming community in northern Ethiopia).
} 


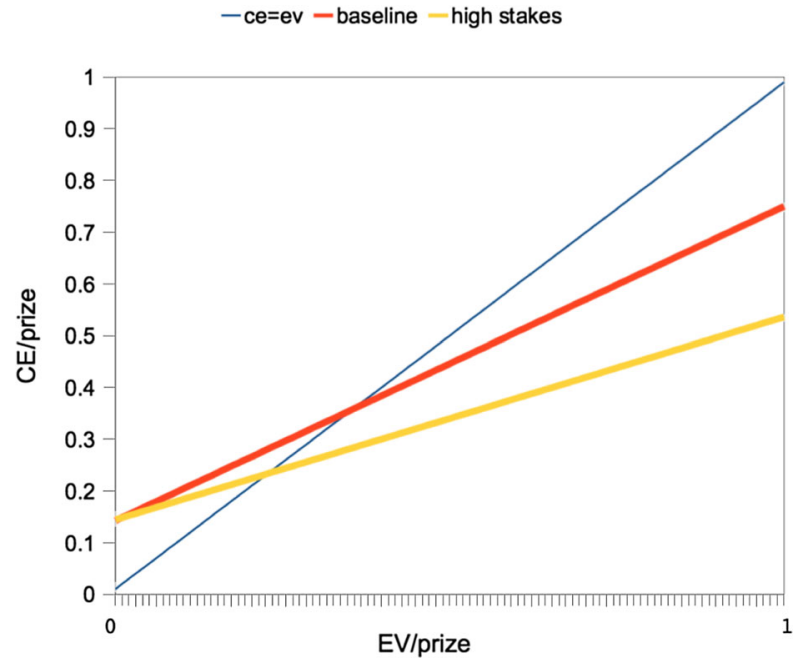

Fig. 4 Ambiguity aversion for gains by stake size

considerably smaller than one $(z=6.12, p<0.001)$, indicating probabilistic insensitivity. This pattern generally corresponds quite well to patterns previously observed (Abdellaoui et al. 2011; Maafi 2011), except that, consistently with what we found under risk, there is no prevalence of global uncertainty aversion $(z=1.52, p=0.13){ }^{4}$

Qualitatively, the pattern we observe under high stakes is similar, with uncertainty seeking for small probabilities $(z=4.64, p<0.001)$, uncertainty aversion for large probabilities $(z=5.90, p<0.001)$, and probabilistic insensitivity $(z=6.01, p<$ $0.001)$. We observe, however, a clear shift downwards as stakes increase. While there is no difference between stake conditions for small probabilities $(z=1.10, p=0.27)$, the difference is highly significant for large probabilities $(z=3.79, p<0.001)$. Once again, this results in a clear difference at the global level $(z=3.98, p<0.001)$, where uncertainty aversion is now the prevalent pattern $(z=5.45, p<0.001)$. The combination of these elements, in turn, also determines a change of the slope of the line, going in the direction of an increase in insensitivity as stakes increase $(z=2.70, p=0.007)$.

In conclusion, we can thus say that the effect of more extreme ambiguity attitudes at the endpoints of the probability scale is mostly driven by stake effects on uncertainty attitudes. For risk, we replicate the previous findings with subjects becoming more risk averse uniformly over the probability spectrum as stakes increase. Under uncertaintyfor which no previous findings on stake effects exist—we find an increase in uncertainty aversion. While attitudes for low probabilities remain unaffected, however, this effect is driven entirely by considerably more uncertainty aversion for large probabilities, ultimately resulting in the rotation of the ambiguity line.

\footnotetext{
${ }^{4}$ For a comparison and correlation analysis of decisions under risk and uncertainty across 30 countries, as well as between gains and losses, see Vieider et al. (2015a).
} 


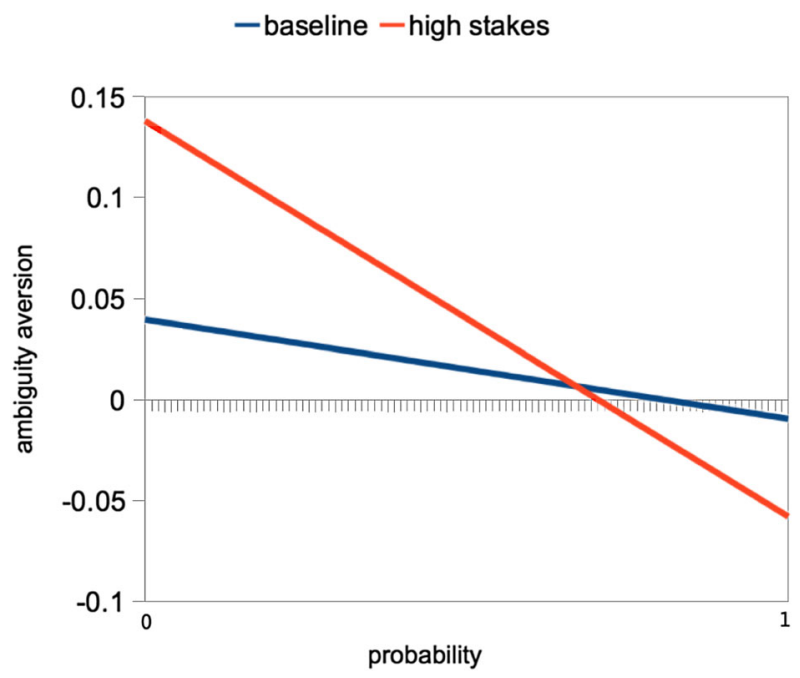

Fig. 5 Ambiguity aversion for losses by stake size

\subsection{Ambiguity attitudes for losses}

We next investigate ambiguity attitudes for losses - the results are depicted in Fig. 5. The first thing that is apparent is that, while ambiguity attitudes in the baseline condition follow a pattern of ambiguity aversion for small probabilities followed by a slight tendency towards ambiguity seeking for moderate-to-large probabilities, these tendencies are again amplified under high stakes. This is, indeed, confirmed by the statistical analysis, with high stakes resulting in increased ambiguity aversion for small probabilities $(z=2.76, p=0.006)$, as well as increased ambiguity seeking for large probabilities $(z=2.20, p=0.028)$ compared to the baseline condition. While these two contrasting effects cancel out at the global level, so that on average, there is no difference $(z=1.16, p=0.25)$, there is once again a clear difference in slopes $(z=2.69, p=0.007)$, indicating more extreme reactions at the endpoints of the probability scale under high stakes, similar to the ones already observed for gains.

Once again, we can trace this effect back to its constituent parts under risk and uncertainty. Figure 6 shows the effect of stake variations on risk attitudes for losses. The general pattern is one of risk aversion for small probabilities $(z=4.11, p<$ $0.001)$ and risk seeking for large probabilities $(z=6.43, p<0.001)$, as well as probabilistic insensitivity $(z=6.36, p<0.001)$. This pattern thus corresponds to what has typically been found in the literature (Abdellaoui 2000). It is furthermore clear that there is no difference between the conditions, either for small probabilities $(z=0.87, p=0.39)$, large probabilities $(z=1.11, p=0.27)$, or globally $(z=$ $0.09, p=0.92)$. It follows that there is also no difference in slopes $(z=0.76, p=$ $0.44)$. This confirms our hypothesis that risk attitudes for losses will not be affected by stake size.

Figure 7 shows the linear regression lines for uncertain losses under the different stakes. The pattern in the baseline condition is again one of uncertainty aversion for 


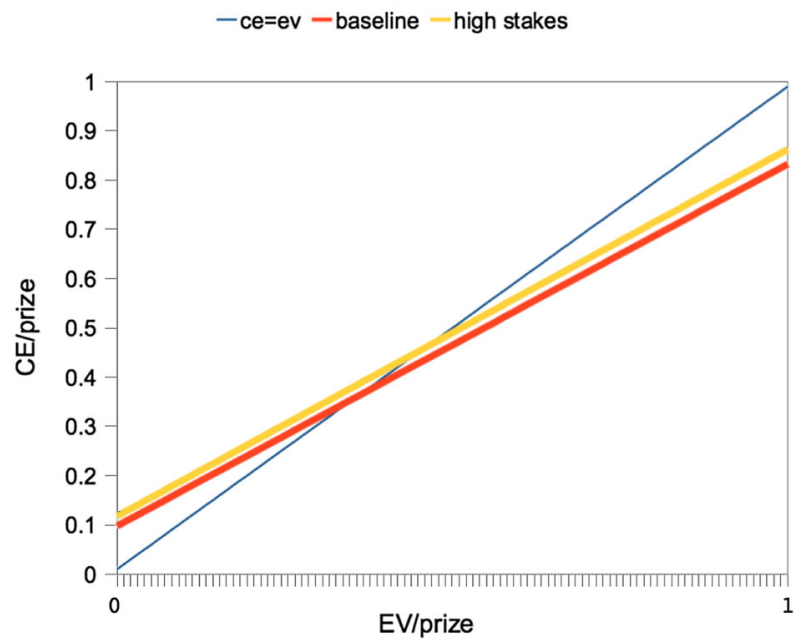

Fig. 6 Ambiguity aversion for gains by stake size

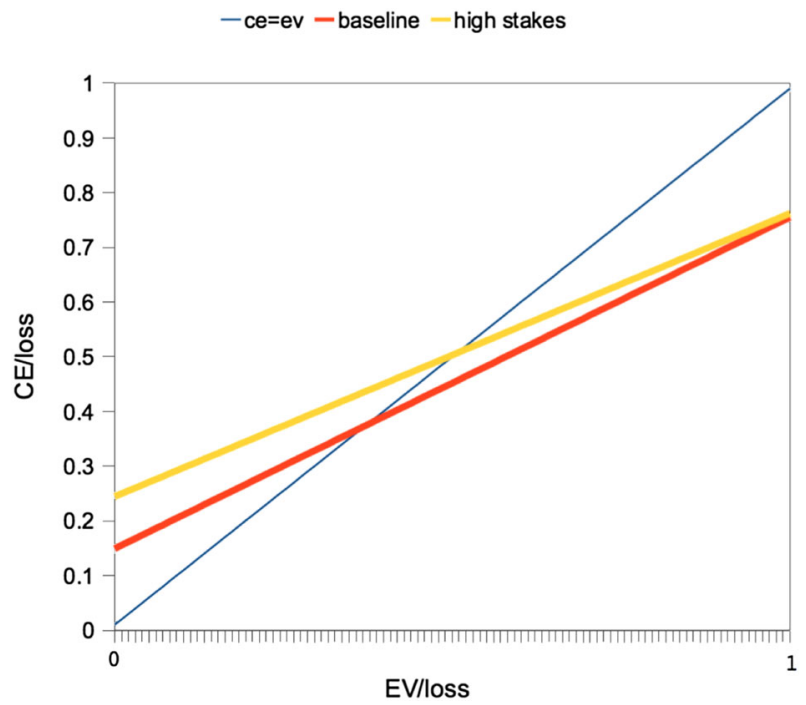

Fig. 7 Ambiguity aversion for gains by stake size

small probabilities $(z=4.11, p<0.001)$ and uncertainty seeking for large probabilities $(z=4.62, p<0.001)$. While there is again significant probabilistic insensitivity ( $z=4.74, p<0.001$ ), globally, there is no prevailing trend, with the uncertainty aversion for small probabilities and uncertainty seeking for large probabilities balancing each other out $(z=0.03, p=0.98)$. Furthermore, there is now a clear stake effect for small probabilities, with increased aversion to uncertainty resulting from higher stakes $(z=2.54, p=0.011)$. While there is no significant effect for large proba- 
bilities $(z=1.00, p=0.32)$ or in global terms $(z=0.88, p=0.38)$, probabilistic insensitivity increases even further under high stakes as compared to the baseline $(z=2.24, p=0.025)$.

We thus find very similar effects in terms of ambiguity attitudes to those already observed in the gain domain. Ambiguity attitudes become more extreme at the endpoints. This effect is driven again mainly by a rotation of the regression line when high stakes are offered under uncertainty. And again, high stakes increase uncertainty aversion, while they do not affect uncertainty seeking in the relevant parts of the probability spectrum.

\section{Discussion}

We investigated the effect of stake variations on ambiguity attitudes over the probability spectrum, for both gains and losses. We then traced these effects back to stake effects separately for risk and uncertainty to determine what may be driving the effects we find in terms of ambiguity attitudes. For risk, we broadly confirmed the previous findings in the literature. In particular, we found risk taking to be reduced or risk aversion to be increased for gains in a clean between subjects design which excludes possible contrast effects. We furthermore show that this reduction in risk seeking is uniform over the probability space. For risky losses, we found no evidence of stake effects. This goes towards further clarifying the previous findings, with, e.g., (Schoemaker 1990) finding no effect of stakes on risk attitudes for losses, while Fehr-Duda et al. (2010) found inconclusive effects of stakes and Bosch-Domènech and Silvestre (2006) found rather strong effects. Vieider (2012) found some effects of stake size on probability weighting functions for losses, which were however counteracted by opposite effects on utility, with the overall effect thus tending towards no difference.

More originally, we provide the first evidence on stake effects for decisions under uncertainty, where the probabilities associated with the different outcomes are unknown or vague. Contrary to our initial tentative hypothesis, effects under uncertainty do not per se tend to be weaker than under risk. Instead, there is a qualitative difference in stake effects that emerges between risk and uncertainty. While the regression lines stay parallel for risk under both gains and losses, indicating an effect that is equally strong over the whole probability space, for uncertainty, we observe a pivoting in the regression line, indicating decreased probabilistic sensitivity as stakes increase-or equivalently, more pronounced reactions at the extremes of the probability distribution. For gains, there is also a significant downward shift over the whole probability space, indicating increased aversion to uncertainty under high stakes overall, which mimics the effect obtained under risk. For losses, there is no global effect, just as under risk. There is, however, the same pivoting effect as observed for gains.

Given the null or parallel effects under risk, the pivoting of the lines under uncertainty indicates differences in ambiguity attitudes over the probability spectrum. We find high stakes to induce increased ambiguity seeking for small probabilities of gains and for moderate-to-large probabilities of losses, and increased ambiguity aversion for small-to-moderate probability losses and moderate-to-high probability gains. This effect can be traced back mostly to the effect of stakes on decisions under uncertainty- 
an issue on which no previous evidence existed. Under uncertainty, people seem to display an even more pronounced possibility effect, induced by a movement from a zero probability to a small positive probability, as well as a more pronounced certainty effect, induced by moving from a large probability to absolute certainty. The insensitivity to probability changes in the intermediate ranges thus increases as stakes increase.

One issue that needs to be addressed explicitly is the extent to which the effects we have found may generalize to other countries, considering that in absolute terms, our Ethiopian subjects were considerably more risk seeking than generally found in western countries. A reassuring indication in this direction derives from the fact that for risk, we were able to replicate the previous findings on stake effects. Indeed, the stake effects on risk we found correspond closely to effects obtained in China (Fehr-Duda et al. 2010), India (Binswanger 1980), the US (Holt and Laury 2002), France (Lefebvre et al. 2010), and the UK (Bouchouicha and Vieider 2016). Given that absolute risk attitudes between these countries have been found to differ (Rieger et al. 2014; L'Haridon and Vieider 2016), while stake effects on risk attitudes were (qualitatively) the same, we believe that our results on stake effects will generalize to different countries.

This opens the floor for the discussion of some potential real-world consequences. Our findings may, indeed, be important for obtaining a better understanding of such processes, to the extent that uncertainty is more realistic and common than risk. Compared, for instance, to studies that have investigated insurance issues with known probability risks, our results show that insurance uptake is likely to be higher than the one found under risk, given ambiguity aversion for small-probability losses. This is even more true for large negative consequences, for which we have seen uncertainty aversion for losses to increase, while risk aversion remained unaffected. While some studies have found a higher propensity to buy insurance for larger probability, lower consequence events (Kunreuther and Pauly 2004; Kunreuther et al. 2001; Slovic et al. 1977), such studies did not generally control for all possible confounds, such as, for instance, the expected value of the loss (in laboratory studies) or the subjective probability of the loss (for real-world evidence; see Laury et al. 2009, for a detailed discussion).

Ambiguity aversion has also been indicated as a possible cause of financial biases, such as the stock market participation puzzle (Cao et al. 2005; Easly and O'Hara 2009). Recently, Dimmock et al. (2015) found that stock market participation, as well as the ownership of small businesses, is negatively correlated with ambiguity-induced insensitivity. To the extent that such slopes become more extreme as stakes increase, this may, indeed, be an important factor for the explanation of such decisions, given the overestimation of small probabilities of losses and underestimation of moderateto-large probabilities of gains that derive from it. The latter point is further reinforced by the fact that for large stakes, we find uncertainty aversion for small-probability losses to be stronger than uncertainty loving for gains of equal probability. Given a symmetric distribution of stock returns about a positive mean, this may by itself imply that people shy away from such investments. 


\section{Conclusion}

The main objective of this paper was to test the effect of stake size on ambiguity attitudes. We found that under high stake ambiguity, attitudes become more extreme, with increased ambiguity seeking for small-probability gains and large-probability losses relative to the baseline condition. Moreover, we also found increased ambiguity aversion for large-probability gains and small-probability losses. We then traced these effects back to stake effects under risk and uncertainty. For risk, we replicated the classic result of increased risk aversion for gains under large stakes. We also brought additional evidence indicating that such risk aversion increases uniformly over the probability space, and that for losses, stakes have no effect on decisions. For uncertainty, we found high stakes to increase global uncertainty aversion for gains and not to affect global uncertainty aversion for losses. For both uncertain gains and losses, however, we found incentives to affect the relative incidence of uncertainty aversion towards the endpoints of the probability scale. In particular, subjects were found to be more uncertainty averse for large-probability gains and small-probability losses under high stakes. Taken together, these findings may help explaining behavior in situations involving small-probability large outcome losses, such as insurance decisions and stock market participation.

Open Access This article is distributed under the terms of the Creative Commons Attribution 4.0 International License (http://creativecommons.org/licenses/by/4.0/), which permits unrestricted use, distribution, and reproduction in any medium, provided you give appropriate credit to the original author(s) and the source, provide a link to the Creative Commons license, and indicate if changes were made.

\section{References}

Abdellaoui, M. (2000). Parameter-free elicitation of utility and probability weighting functions. Management Science, 46(11), 1497-1512.

Abdellaoui, M., Baillon, A., Placido, L., \& Wakker, P. P. (2011). The rich domain of uncertainty: source functions and their experimental implementation. American Economic Review, 101, 695-723.

Abdellaoui, M., Vossmann, F., \& Weber, M. (2005). Choice-based elicitation and decomposition of decision weights for gains and losses under uncertainty. Management Science, 51(9), 1384-1399.

Abdellaoui, M., L'Haridon, O., \& Zank, H. (2010). Separating curvature and elevation: a parametric probability weighting function. Journal of Risk and Uncertainty, 41(1), 39-65.

Akay, A., Martinsson, P., Medhin, H., \& Trautmann, S. (2012). Attitudes toward uncertainty among the poor: an experiment in rural Ethiopia. Theory and Decision, 73(3), 453-464.

Binswanger, H. P. (1980). Attitudes toward risk: experimental measurement in rural India. American Journal of Agricultural Economics, 62(3), 395-407.

Birnbaum, M. H. (1999). Testing critical properties of decision making on the internet. Psychological Science, 10(5), 399-407.

Bosch-Domènech, A., \& Silvestre, J. (2006). Reflections on gains and losses: A 2 x 2 x 7 experiment. Journal of Risk and Uncertainty, 33, 217-235.

Bouchouicha, R., \& Vieider, F. M. (2016) 'Accommodating changes in risk preferences over probabilities and stakes under prospect theory' (Working Paper)

Cao, H. H., Wang, T., \& Zhang, H. H. (2005). Model uncertainty, limited market participation, and asset prices. Review of Financial Studies, 18(4), 1219-1251.

Chateauneuf, A., Eichberger, J., \& Grant, S. (2007). Choice under uncertainty with the best and worst in mind: neo-additive capacities. Journal of Economic Theory, 137(1), 538-567. 
Dimmock, S. G., Kouwenberg, R., \& Wakker, P. P. (2015). Ambiguity attitudes in a large representative sample. Management Science, 62(5), 1363-1380.

Doerr, U., Toman, O. M., \& Schmidt, U. (2011) 'Overconfidence and Risk Management of Ethiopian Farmers.' University of Kiel (Working paper)

Easly, D., \& O'Hara, M. (2009). Ambiguity and nonparticipation: the role of regulation. Review of Financial Studies, 22(5), 1817-1843.

Ellsberg, D. (1961). Risk, ambiguity and the savage axioms. Quarterly Journal of Economics, 75(4), 643669.

Etchart-Vincent, N. (2004). Is probability weighting sensitive to the magnitude of consequences? An experimental investigation on losses. Journal of Risk and Uncertainty, 28(3), 217-235.

Etchart-Vincent, N., \& L'Haridon, O. (2011). Monetary incentives in the loss domain and behavior toward risk: an experimental comparison of three reward schemes inclusing real losses. Journal of Risk and Uncertainty, 42, 61-83.

Fehr-Duda, H., Bruhin, A., Epper, T. F., \& Schubert, R. (2010). Rationality on the rise: why relative risk aversion increases with stake size. Journal of Risk and Uncertainty, 40(2), 147-180.

Fox, C. R., \& Tversky, A. (1998). A belief-based account of decision under uncertainty. Management Science, 44(7), 879-895.

Greenwald, A. G. (1978). Within-subject designs: to use or not to use? Psychological Bulletin, 82(1), 314-320.

Henrich, J., \& McElreath, R. (2002). Are peasants risk averse decision makers? Current Anthropology, 43(1), 172-181.

Hogarth, R. M., \& Einhorn, H. J. (1990). Venture theory: a model of decision weights. Management Science, 36(7), 780-803.

Holt, C. A., \& Laury, S. K. (2002). Risk aversion and incentive effects. American Economic Review, 92(5), 1644-1655.

Kachelmeier, S. J., \& Shehata, M. (1992). Examining risk preferences under high monetary incentives: experimental evidence from the People's Republic of China. American Economic Review, 82(5), 1120-1141.

Kahneman, D., \& Tversky, A. (1979). Prospect theory: an analysis of decision under risk. Econometrica, 47(2), 263-291.

Kilka, M., \& Weber, M. (2000). Home bias in international stock return expectation. Journal of Psychology and Financial Markets, 1(3-4), 176-192.

Kunreuther, H., \& Pauly, M. (2004). Neglecting disaster: why don't people insure against large losses? Journal of Risk and Uncertainty, 28(1), 5-21.

Kunreuther, H., Novemsky, N., \& Kahneman, D. (2001). Making low probabilities useful. Journal of Risk and Uncertainty, 23, 103-120.

Laury, S. K., McInnes, M. M., \& Todd Swarthout, J. (2009). Insurance decisions for low-probability losses. Journal of Risk and Uncertainty, 39, 17-44.

Lefebvre, M., Vieider, F. M., \& Villeval, M. C. (2010). Incentive effects on risk attitude in small probability prospects. Economics Letters, 119, 115-120.

L'Haridon, O., \& Vieider, F. M. (2016). 'All over the map: heterogeneity of risk preferences across individuals, contexts, and countries.' EM-DP2016-04, University of Reading.

Maafi, H. (2011). Preference reversals under ambiguity. Management Science, 57(11), 2054-2066.

Muthukrishnan, A. V., Wathieu, L., \& Jing Alison, X. (2009). Ambiguity aversion and the preference for established brands. Management Science, 55(12), 1933-1941.

Read, D. (2005). 'Monetary incentives, what are they good for?' Journal of Economic Methodology, 12, $265-276$

Rieger, M. O., Wang, M., \& Hens, T. (2014). Risk preferences around the world. Management Science, 61(3), 637-648.

Ritov, I., \& Baron, J. (1990). 'Reluctance to vaccinate: omission bias and ambiguity.' Journal of Behavioral Decision Making, 3, 263-277

Savage, L. J. (1954). The foundations of statistics. New York: Wiley.

Schoemaker, P. J. H. (1990). Are risk-attitudes related across domains and response modes? Management Science, 36(12), 1451-1463.

Scholten, M., \& Read, D. (2014). Prospect theory and the "forgotten" fourfold pattern of risk preferences. Journal of Risk and Uncertainty, 48(1), 67-83. 
Slovic, P., Fischhoff, B., Lichtenstein, S., Corrigan, B., \& Combs, B. (1977). Preference for Insuring against probably small losses: insurance implications. Journal of Risk and Insurance, 44(2), 237-257.

Starmer, C. (2000). Developments in non-expected utility theory: the hunt for a descriptive theory of choice under risk. Journal of Economic Literature, 38, 332-382.

Trautmann, S. T., \& van de Kuilen, G. (2015). 'Ambiguity attitudes.' In 'The Wiley Blackwell Handbook of Judgment and Decision Making'. New York :Wiley

Trautmann, S. T., Vieider, F. M., \& Wakker, P. P. (2011). Preference reversals for ambiguity aversion. Management Science, 57(5), 1320-1333.

Tversky, A., \& Kahneman, D. (1992). Advances in prospect theory: cumulative representation of uncertainty. Journal of Risk and Uncertainty, 5, 297-323.

van de Kuilen, G., \& Wakker, P. P. (2011). The midweight method to measure attitudes toward risk and ambiguity. Management Science, 57, 582-598.

Vieider, F. M. (2011). Separating real incentives and accountability. Experimental Economics, 14(4), 507518.

Vieider, F. M. (2012). Moderate stake variations for risk and uncertainty, gains and losses: methodological implications for comparative studies. Economics Letters, 117, 718-721.

Vieider, F. M., Beyene, A., Bluffstone, R. A., Dissanayake, S., Gebreegziabher, Z., Martinsson, P., \& Mekonnen, A. (2016). 'Measuring risk preferences in rural Ethiopia.' Economic Development and Cultural Change (forthcoming)

Vieider, F. M., Lefebvre, M., Bouchouicha, R., Chmura, T., Hakimov, R., Krawczyk, M., et al. (2015a). Common components of risk and uncertainty attitudes across contexts and domains: evidence from 30 countries. Journal of the European Economic Association, 13(3), 421-452.

Vieider, F. M., Chmura, T., Fisher, T., Kusakawa, T., Martinsson, P., Thompson, F. M., et al. (2015b). Withinversus between-country differences in risk attitudes: implications for cultural comparisons. Theory and Decision, 78(2), 209-218.

Wakker, P. P. (2010). Prospect theory for risk and ambiguity. Cambridge: Cambridge University Press. 